\title{
Late-time hohlraum pressure dynamics in supernova remnant experiments
}

\author{
O. A. Hurricane, ${ }^{\text {a) }}$ S. G. Glendinning, and B. A. Remington \\ University of California, Lawrence Livermore National Laboratory, P.O. Box 808, Livermore, \\ California 94550
}

\author{
R. P. Drake and K. K. Dannenberg \\ University of Michigan, Department of Atmospheric, Oceanic, and Space Sciences, \\ Space Research Building, 2455 Hayward, Ann Arbor, Michigan 48109-2143
}

(Received 11 December 2000; accepted 29 March 2001)

\begin{abstract}
It is shown that laser driven hohlraums obtain significant internal pressures which affect the hydrodynamics of high-energy density shock-tube experiments. By incorporating this previously neglected hohlraum pressure effect (in addition to the usual x-ray drive) into computer simulations which model the NOVA laser driven supernova remnant experiment [R. P. Drake, S. G. Glendinning, K. Estabrook, B. A. Remington, R. McCray, R. J. Williams, L. J. Suter, T. B. Smith, J. J. Carroll III, R. A. London, and E. Liang, Phys. Rev. Lett. 81, 2068 (1998)], calculations are able to reproduce the observed structure of hydrodynamic features. (C) 2001 American Institute of Physics. [DOI: 10.1063/1.1373416]
\end{abstract}

The NOVA ${ }^{1}$ laser facility has been used to explore the physics of high-energy density plasmas for a number of years. Areas of interest include hydrodynamics of high Mach number shocks as well as Richtmyer-Meshkov (RM) and Rayleigh-Taylor (RT) instabilities, ${ }^{2}$ radiation flow, ${ }^{3}$ and equations of state-areas which have application to inertial fusion and astrophysics. The National Ignition Facility $(\mathrm{NIF}),{ }^{4}$ once complete, will carry on the tradition of these high-energy density plasma studies.

Experiments in the NOVA (and future NIF) facility often involve attaching a small, usually cylindrical, target package to a hole in the side of a much larger gold cylinder [a hohlraum; see Fig. 1(a)]. When illuminated on the inner surface using high power lasers, the hohlraum becomes a source of a fairly uniform field of $x$ rays which drive the target package. In the past, the hohlraum was thought to couple energy to the target package only through the $\mathrm{x}$ rays, but discrepancies between data of recent long lived hydrodynamic experiments and simulations suggests otherwise. ${ }^{5-7}$

Recent work has shown that by adding the effect of gold plasma pressure, simulations can be brought into agreement with the experimental data. ${ }^{8}$ In this letter we report our work which involves modeling the effects of the hohlraum pressure and our success in resolving discrepancies between simulation and experimental results for the supernova remnant (SNR) experiment (Fig. 1). ${ }^{5,7,9}$

In the SNR experiment, hohlraum $x$ rays, with a radiation temperature of $\sim 220 \mathrm{eV}$, ablate a $200 \mu \mathrm{m}$ thick $2 \%$ brominated plastic $\left(\mathrm{C}_{50} \mathrm{H}_{48} \mathrm{Br}_{2}\right)$ plug of density $\rho=1.22$ $\mathrm{g} / \mathrm{cm}^{3}$ which is held to the hohlraum with a Au washer (Fig. 1). The $x$ rays drive a strong shock (peak pressure of $\sim 50$ $\mathrm{Mb}$ ) through the plug. When the shock "breaks out" of the end of the plug, the material is ejected across a $150 \mu \mathrm{m}$ gap and a rarefaction wave traveling back towards the hohlraum

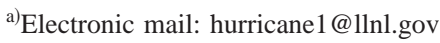

is created which expands the shocked plug material. The ejected plug material provides a flow of plasma which impacts the end of a cylinder of low density, $\rho=0.048 \mathrm{~g} / \mathrm{cm}^{3}$ carbon resorcinol foam. Upon impact, a shock is transmitted into the foam and the foam compresses. As the foam compresses, perturbations of wavelength $\lambda=100 \mu \mathrm{m}$ and a peak-valley amplitude of $4 \mu \mathrm{m}$ that were etched into the foam surface begin to grow under the action of a RM like instability at the contact discontinuity between the plug ejecta and the foam. At late times, $\sim 7-10 \mathrm{~ns}$, residual plug material is propelled out of the Au washer in a distinct compressed feature we call the plug remnant.

LASNEX $^{10}$ simulations, described in Ref. 5, successfully reproduced the time the ablative shock broke out of the plug and the qualitative structure of the forward shock and contact discontinuity region. Quantitatively, the forward shock in the simulation was too small (84 versus $107 \mathrm{~km} / \mathrm{s}$ ) for experiments using $40 \mathrm{mg} / \mathrm{cc} \mathrm{SiO}_{2}$ foam. In addition, one qualitative feature was not predicted correctly. This was the presence of the plug remnant. ${ }^{7}$ Data from the experiment show that at late times the plug remnant experienced a change in velocity, going from $\sim 52$ to $\sim 82 \mathrm{~km} / \mathrm{s}$, causing it to compress against the contact discontinuity as shown in Fig. 1(b). The early simulations of this experiment predicted no such acceleration. We offer that the discrepancies of the plug remnant dynamics as seen in the experiments and simulations are due to a previously neglected effect of Au plasma pressure development inside the hohlraum. ${ }^{8}$

Imagine that the plug remnant can be considered a disk of material with thickness, $H$, and density, $\rho$, we can estimate the time-averaged pressure required to deliver a change in velocity $(\Delta v)$ to the plug using Newton's law

$$
\langle P\rangle \sim \frac{\rho H \triangle v}{\triangle t},
$$

where $\triangle t \sim L /\langle v\rangle$ is the interaction time during which the 


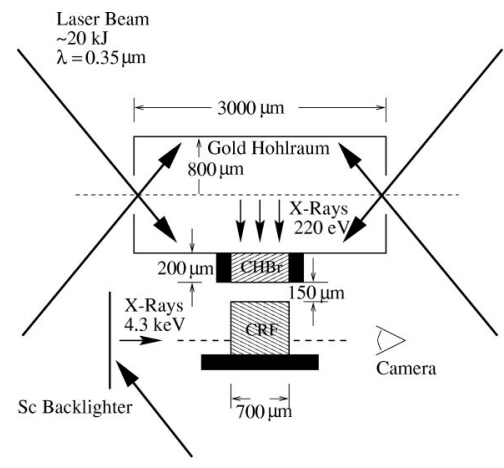

(a)

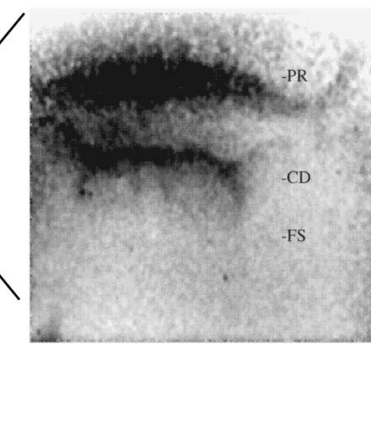

(b)
FIG. 1. (a) A typical Nova Scale 1.0 high-energy density experimental configuration (dimensions and materials shown are those of the SNR experiment) (Refs. 5 and 7). X rays from a laser heated backlighter foil are used to create an image (a radiograph) of the target. A radiograph, taken at $t=9.8$ $\mathrm{ns}$, for the SNR experiment is shown in (b). The forward shock (FS), contact discontinuity (CD), and plug remnant (PR) features are labeled.

plug is propelled down a cylinder of length, $L$, of constant radius at mean speed $\langle v\rangle$ (the cylinder in mind here being the $\mathrm{Au}$ washer that holds the plastic plug). Assume that once the plug remnant is expelled from the cylinder, it obtains a constant speed and that while it is accelerated its mass is constant. From the SNR experiment, the $2 \% \mathrm{Br}$ plug is observed to have $\Delta v \sim 30 \mathrm{~km} / \mathrm{s},\langle v\rangle \sim 67 \mathrm{~km} / \mathrm{s}, \rho \sim 1.5 \mathrm{~g} / \mathrm{cm}^{3}, H$ $\sim 100 \mu \mathrm{m}$, and $L \sim 200 \mu \mathrm{m}$, thus $\Delta t \sim 3 \mathrm{~ns}$ and $\langle P\rangle \sim 15 \mathrm{Mb}$, a surprisingly large pressure.

Using scaling formulas presented in Lindl, ${ }^{2}$ we can construct a simple analytic model which suggests how a large hohlraum pressure develops and is maintained if we neglect hydrodynamics occurring inside the hohlraum: The mass per unit area $\left(m_{\mathrm{Au}}\right)$ of $\mathrm{Au}$ ablated from a hohlraum wall when subjected to radiation of temperature $T_{r}$ for a period of time $\tau$ is $m_{\mathrm{Au}}\left(\mathrm{g} / \mathrm{cm}^{2}\right) \sim 1.0 \times 10^{-3} T_{r}^{1.86}\left(10^{2} \mathrm{eV}\right) \tau^{0.75}$ (ns), where the units of measure are given in parenthesis. $\mathrm{Au}$ atoms ejected from the hohlraum wall ionize to a degree approximated by $\widetilde{Z} \sim 23 T_{r}^{0.45}\left(10^{2} \mathrm{eV}\right)$. The number of plasma particles per unit area released from the hohlraum wall is then $N\left(\mathrm{~cm}^{-2}\right) \sim 7.4 \times 10^{19} T_{r}^{2.3}\left(10^{2} \mathrm{eV}\right) \tau^{0.75}$ (ns). Assuming that the hohlraum is a long cylinder of radius $R$, volume-averaged plasma particle pressure is estimated to be

$$
\bar{P}(\mathrm{Mb}) \sim 2.4 \times 10^{-2} T_{r}^{3.3}\left(10^{2} \mathrm{eV}\right) \tau^{0.75}(\mathrm{~ns}) / \mathrm{R}(\mathrm{cm})
$$

assuming thermodynamic equilibrium. For the SNR experiment $R \sim 0.08 \mathrm{~cm}$ and $T_{r} \sim 2.2 \times 10^{2} \mathrm{eV}$ at $\tau=1 \mathrm{~ns}$, so $\bar{P}$ $\sim 4.1 \mathrm{Mb}$ which is significant. A slightly more precise estimate of the material pressure is shown in Fig. 2, where a Thomas-Fermi ionization model is used to calculate $\widetilde{Z}$ instead of the formula given above; the results do not differ greatly from the estimate written above. In Fig. 2, after $1 \mathrm{~ns}$ the pressure is nearly constant in time, an effect due to the cooling of the plasma in time being essentially offset by the continual addition of particles to the hohlraum. The radiation temperature history shown in Fig. 2 comes from LASNEX simulations of the hohlraum. ${ }^{11}$ [The above estimates assume that radiation is evenly applied to the inside of the hohlraum wall. If the same amount of radiant energy were concentrated

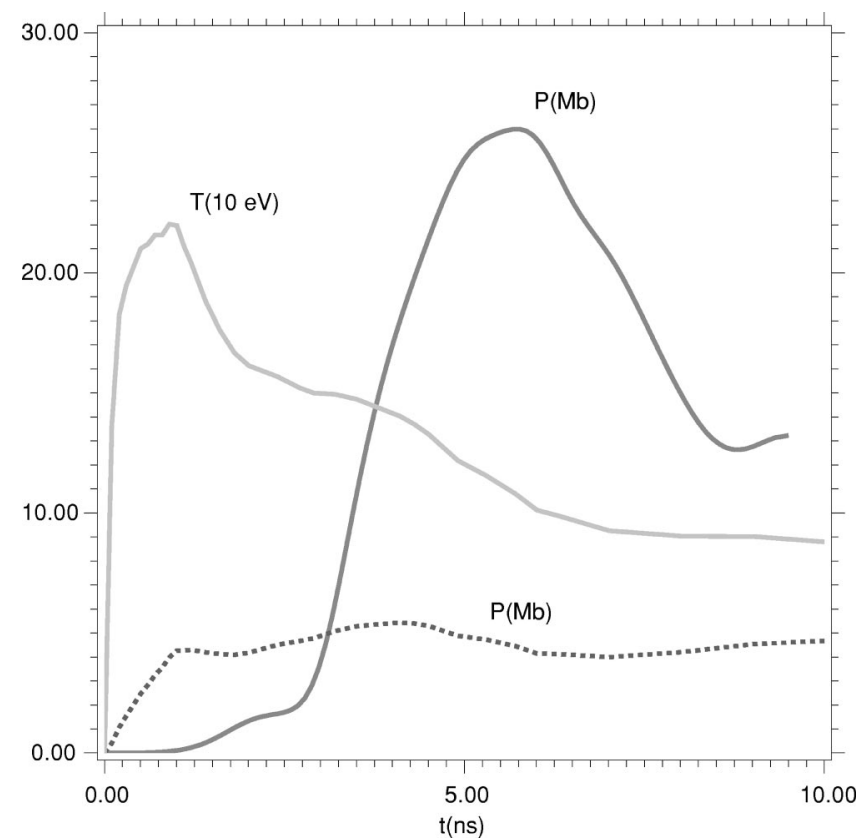

FIG. 2. The dotted line is $\bar{P}$ from Eq. (2). The light solid curve labeled $T$ is the hohlraum radiation temperature pulse shape taken from the SNR experiment which is used to calculate $\bar{P}$ as well as drive the CALE simulations. The dark solid curve is the pressure history at the plastic-Au interface calculated from a 2D hohlraum-package simulation discussed below.

into a small subarea of the hohlraum wall (i.e., a hot spot) then the matter ablation rate from the walls would be reduced by a factor of $\left(A_{\mathrm{hs}} / A_{w}\right)^{0.425}$, where $A_{\mathrm{hs}}$ is the area of the hot spots and $A_{w}$ is the area of the hohlraum wall.]

In addition to the burnoff and ionization effect described above, hydrodynamical actions inside the hohlraum also contribute to the effective pressure on the plug remnant (e.g., the stagnation pressure caused by Au converging on the hohlraum axis or the creation of jets of material). Simulations which attempt to model the fully three-dimensional (3D) hohlraum-package system have been performed in two dimensions (2D), by treating the hohlraum as an infinitely long cylinder and the experimental package as a slab (see Fig. 3).

The 2D C-based arbitrary Lagrangian-Eulerian (CALE) radiation hydrodynamics simulations ${ }^{12}$ which incorporate the hohlraum show that the Au plasma ablated from the inner walls of the hohlraum never actually stagnates on the axis since plasma ablated from the plug (blowoff) reaches the axis first. Tabulated material equations of state and opacity tables are used in this simulation and in the simulations discussed below. The radiation temperature drive used in the simulation is shown in Fig. 2. Figure 2 also shows the pressure of the Au-plastic Lagrangian boundary along the symmetry line of the simulation. A peak pressure of $P \sim 27 \mathrm{Mb}$ is achieved. The curve labeled hohlraum in Fig. 4 shows the plug trajectory calculated using the $2 \mathrm{D}$ hohlraum simulation. Although the time average pressure at the contact boundary calculated from the simulation $(\langle P\rangle \sim 13 \mathrm{Mb})$ is only indirectly related to the ablation pressure it is reassuring to note that it is in good agreement (within 13\%) with the estimate made using the experimental data and Eq. (1). The volume average pressure computed from the simulation $(\bar{P}=4.0$ 


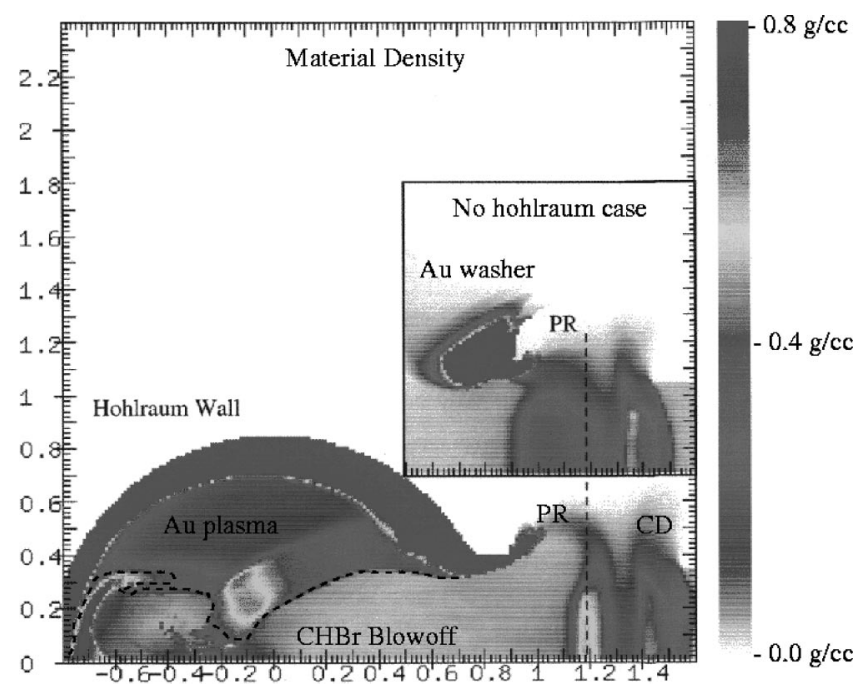

FIG. 3. The effect of the hohlraum on the plug remnant position at $9.5 \mathrm{~ns}$. The boundary between the $\mathrm{CHBr}$ blowoff and Au plasma in the hohlraum has been highlighted with a dashed line. The inset shows the density features of a comparison simulation that does not incorporate a hohlraum or the effects of hohlraum pressure as a boundary condition. The plug-remnant $(\mathrm{PR})$ and contact discontinuity features $(\mathrm{CD})$ in both simulations are labeled. The spatial units are in $10^{3} \mu \mathrm{m}$.

$\pm 0.2 \mathrm{Mb}$ after $t=1 \mathrm{~ns}$ ) is in very good agreement (within $2.5 \%$ ) with that calculated a priori from Eq. (2). (In appreciating the above comparisons, one must keep in mind that the simulation does not properly represent the interpenetration of nearly collisionless plasmas that one would associate with hot low-density ablated material.)

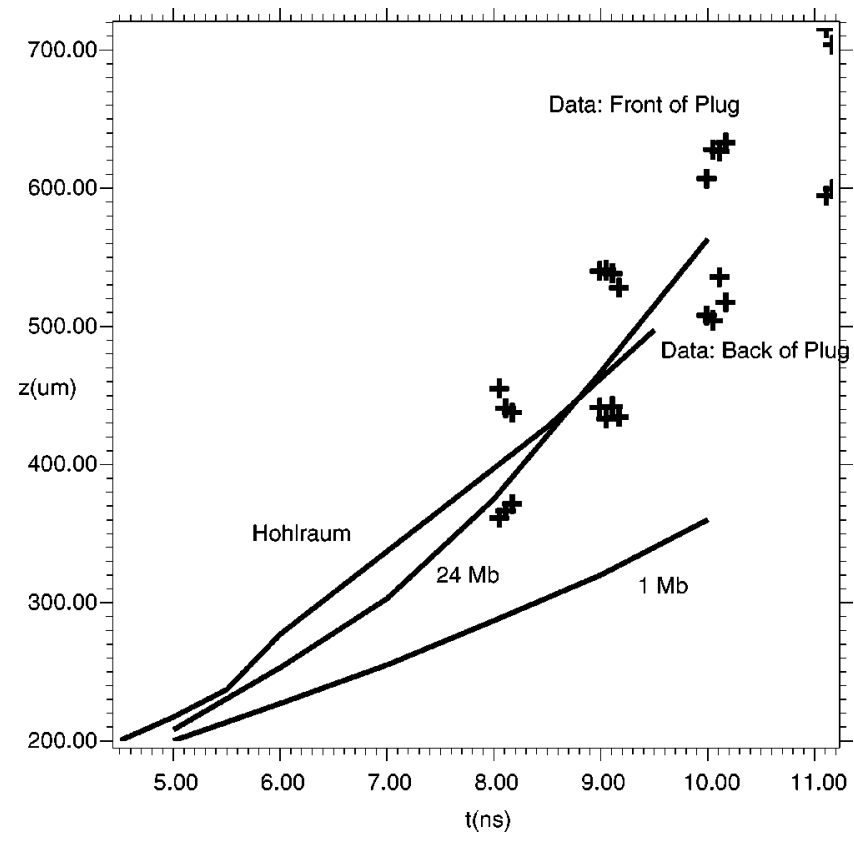

FIG. 4. Shown are the trajectories of the density peak of the plug remnant verses time as computed with CALE. Using the $24 \mathrm{Mb}$ pressure boundary condition, the trajectory of the plug remnant is bracketed by data on the position of the front and back of the plug (defined as being $75 \%$ of the peak density). Another trajectory using a hohlraum pressure of $1 \mathrm{Mb}$ is shown for comparison. The trajectory labeled "hohlraum" comes from the 2D hohlraum simulation shown in Fig. 3.
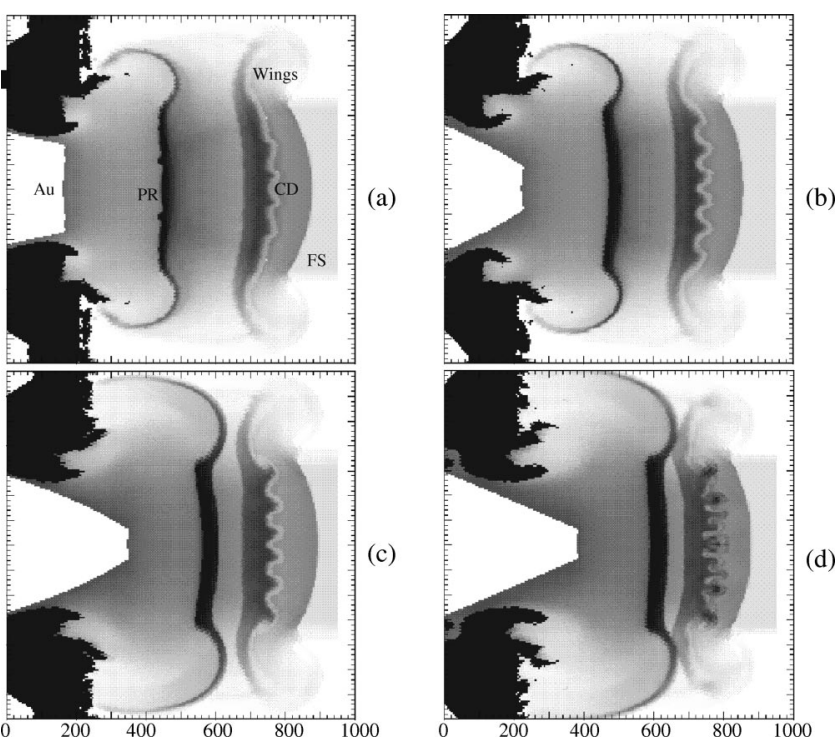

FIG. 5. The figure shows calculated radiographs for CALE simulations at $t=10 \mathrm{~ns}$ using a hohlraum pressure boundary condition of $20 \mathrm{Mb}$ (a), $22 \mathrm{Mb}$ (b), $24 \mathrm{Mb}$ (c), and $25 \mathrm{Mb}$ (d). In (a) the critical features are labeled in a way similar to Fig. 1(b). The hohlraum pressure affects the spacing of features and the degree of structure in the instability occurring at the contact discontinuity. Frame (c) appears to best match the position of the plug remnant. Here the hohlraum would occupy the space to the left. Each image is $10^{3} \mu \mathrm{m}$ (horizontal) by $1.4 \times 10^{3} \mu \mathrm{m}$ (vertical).

The CALE code has also been used to examine the effect of hohlraum pressure on the detailed fine-structure of the unstable interface (see Fig. 5). In the simulation, the x-ray radiation is treated as a time dependent temperature source (see $T$ shown in Fig. 2) on the left most boundary. Fixed boundary conditions were applied to all sides of the simulation domain except for the left boundary which was free. The hohlraum pressure was applied as a boundary condition on the radiation drive (left) side of the experimental package.

Figure 5 shows simulated radiographs of the target package at $t=10 \mathrm{~ns}$ using four different values of the average hohlraum pressure. The pressures used range from 20 to 25 $\mathrm{Mb}$. Fast-Fourier transforms (FFTs) of the contact surface seen in the experimental radiographic image show that the dominant wave numbers correspond to wavelengths with 100,200 , and $50 \mu \mathrm{m}$ with relative spectral powers of 1.0 , 0.8 , and 0.6, respectively. An irregular spectrum at higher wave numbers is also present in the experimental radiograph. The simulations shown in Figs. 5(c) and particularly 5(d) reproduce the long wavelength $(>50 \mu \mathrm{m})$ behavior, but do not reproduce the irregular spectrum at shorter wavelengths. (It is interesting to note that the gross perturbation amplitude is linearly related, with negative slope, to the plug remnantinterface spacing as seen in Fig. 5).

Measurement of the spike position minus bubble position taken at 50\% optical depth gives a measure of the instability amplitude. For the SNR experiment at $t=9.8 \mathrm{~ns}$ the amplitude was $\sim 50 \mu \mathrm{m}$ while it was 36,50 , and $62 \mu \mathrm{m}$ for the calculations shown in Figs. 5(b), 5(c), and 5(d), respectively. Finally, the trajectory of the plug remnant observed in the $24 \mathrm{Mb}$ constant boundary condition simulation (see Fig. 4) is very close to that observed from the full hohlraum 
simulation discussed above. Overall, Fig. 5(c) provides the best match to the experimental data.

To examine whether the absence of a beryllium shocktube cause the SNR target to be more sensitive to hohlraum pressure effects than more typical NOVA shock-tube experiments, simulations of the SNR target that include a hypothetical beryllium sleeve have been performed. These simulations showed that the modeled hohlraum pressure affected the hydrodynamic features in a way identical to that discussed above. We conclude that although the SNR experiment exhibits more decompression than a typical NOVA shock tube experiment, it is not overly sensitive to hohlraum pressure effects. That is, all late-time side-on radiography experiments, with or without a Be tube, may be affected by this hohlraum plasma pressure effect.

In this Letter we have shown that by modeling the hohlraum plasma pressure we are able to greatly improve the degree to which simulations reproduce the hydrodynamic features observed in the SNR experiment. Although it has long been known that hohlraums fill with plasma, the tamping effect of the plasma pressure has never been considered important. Our simulation results suggest that both the gross positions of hydrodynamic features as well as the fine structure of the unstable interface are affected by the hohlraum pressure development. Large plasma pressures which exert a significant force on the experimental package do exist and are consistent with $2 \mathrm{D}$ simulations which attempt to model the complete hohlraum-package system. It is likely that direct simulations that allow fully 3D geometries (e.g., Ref. 8) can clarify the complex dynamical nature of hohlraum pressure development. The influence of hohlraum pressure is important for correctly analyzing old NOVA experiments which last for more than $5 \mathrm{~ns}$ and for future experiments that will take place in the NIF facility since some experiments proposed for NIF may run for as much as $100 \mathrm{~ns}$.

\section{ACKNOWLEDGMENTS}

This work has benefited from conversations with Dr. J. Edwards, Dr. T. Perry, and Dr. P. Stry.

This work was performed under the auspices of the U.S. Department of Energy by the University of California Lawrence Livermore National Laboratory under Contract No. W-7405-Eng-48.

${ }^{1}$ E. M. Campbell, J. T. Hunt, E. S. Bliss, D. R. Speck, and R. P. Drake, Rev. Sci. Instrum. 57, 2101 (1986).

${ }^{2}$ G. Dimonte and B. A. Remington, Phys. Rev. Lett. 70, 1806 (1993).

${ }^{3}$ P. Amendt, S. G. Glendinning, B. A. Hammel et al., Phys. Rev. Lett. 77, 3815 (1996).

${ }^{4}$ J. Lindl, Phys. Plasmas 2, 3933 (1995).

${ }^{5}$ R. P. Drake, S. G. Glendinning, K. Estabrook, B. A. Remington, R. McCray, R. J. Williams, L. J. Suter, T. B. Smith, J. J. Carroll III, R. A. London, and E. Liang, Phys. Rev. Lett. 81, 2068 (1998).

${ }^{6}$ L. M. Logory, P. L. Miller, and P. E. Stry, Astrophys. J., Suppl. 127, 423 (2000).

${ }^{7}$ R. P. Drake, J. J. Carroll III, T. B. Smith, P. Keiter, S. G. Glendinning, O. Hurricane, K. Estabrook, D. D. Ryutov, B. A. Remington, R. Wallace, E. Michael, and R. McCray, Phys. Plasmas 7, 2142 (2000).

${ }^{8}$ B. P. Wilde, M. L. Gittings, T. S. Perry, J. M. Foster, P. A. Rosen, M. Fell, and J. A. Cobble, Bull. Am. Phys. Soc. 44, 39 (1999).

${ }^{9}$ R. P. Drake, J. J. Carroll, K. Estabrook, S. G. Glendinning, B. Remington, and R. McCray, Astrophys. J. Lett. 500, L157 (1998).

${ }^{10}$ G. B. Zimmerman and W. L. Kruer, Comments Plasma Phys. Control. Fusion 2, 51 (1975)

${ }^{11}$ E. Dattolo, L. Suter, M.-C. Monteil, J.-P. Jadaud, N. Dague, S. Glenzer, R. Turner, D. Jaraszek, B. Lasinski, C. Decker, O. Landen, and B. MacGowan, Phys. Plasmas 8, 260 (2001).

${ }^{12}$ R. T. Barton, in Numerical Astrophysics, edited by J. M. Centrella, J. M. LeBlanc, and R. L. Bowers (Jones and Bartlett, Boston, 2000), pp. $482-497$. 